\title{
Constructions of thin-tall Boolean spaces
}

\author{
Juan Carlos MARTínEZ \\ Facultat de Matemàtiques \\ Universitat de Barcelona \\ Gran Via 585 \\ 08007 Barcelona, Spain \\ martinez@ub.edu
}

Recibido: 7 de Noviembre de 2002

Aceptado: 27 de Mayo de 2003

\section{ABSTRACT}

This is an expository paper about constructions of locally compact, Hausdorff, scattered spaces whose Cantor-Bendixson height has cardinality greater than their Cantor-Bendixson width.

2000 Mathematics Subject Classification: 54A25, 54A35, 54G12,03E05, 06E15.

Key words: Scattered space, thin-tall, partitions, $\Delta$-functions, walks, forcing, constructibility

\section{Introduction}

The terminology used in this paper is standard. Undefined set-theoretic terms can be found in [6] or [12]. Undefined topological terms can be found in [5].

The Cantor-Bendixson process for topological spaces is defined as follows. Suppose that $X$ is a topological space. Then, for every ordinal $\alpha$ we define the $\alpha$-derivative of $X$ by: $X^{0}=X$; if $\alpha=\beta+1, X^{\alpha}$ is the set of accumulation points of $X^{\beta}$; and if $\alpha$ is a limit, $X^{\alpha}=\bigcap\left\{X^{\beta}: \beta<\alpha\right\}$. We say that $X$ is scattered, if $X^{\alpha}=\emptyset$ for some ordinal $\alpha$.

The Cantor-Bendixson process permits us to split a scattered space into levels. Suppose that $X$ is a scattered space. We define the height of $X$ by

$$
\operatorname{ht}(X)=\text { the least ordinal } \alpha \text { such that } X^{\alpha} \text { is finite. }
$$

For $\alpha<\operatorname{ht}(X)$, we write $I_{\alpha}(X)=X^{\alpha} \backslash X^{\alpha+1}$. If $x \in I_{\alpha}(X)$, we say that $\alpha$ is the level of $x$ and we write $\rho(x)=\alpha$. Note that $\rho(x)=\alpha$ means that $x$ is an

\footnotetext{
${ }^{1}$ The preparation of this paper was supported by DGI Project BFM2002-01034.
} 
accumulation point of $I_{\beta}(X)$ for any $\beta<\alpha$ but $x$ is not an accumulation point of $X^{\alpha}=\bigcup\left\{I_{\beta}(X): \beta \geq \alpha\right\}$. Then, it is natural to define the width of $X$ as

$$
\operatorname{wd}(X)=\sup \left\{\left|I_{\alpha}(X)\right|: \alpha<\operatorname{ht}(X)\right\} .
$$

Assume that $X$ is a scattered space, $x \in X$ and $U$ is a neighbourhood of $x$. We say that $U$ is a cone on $x$, if $x$ is the only point in $\mathrm{U}$ of level $\geq \rho(x)$. Clearly, if $\mathcal{U}$ is a neighbourhood basis of $x$, we may assume that every $U \in \mathcal{U}$ is a cone on $x$.

Here is an example. Suppose that $\alpha$ is an infinite ordinal. We denote by $T(\alpha)$ the ordinal $\alpha$ equipped with the order topology. Then, if we put $X=T(\alpha)$ we have that $I_{0}(X)$ is the set of all non-limit ordinals of $\alpha$ and for $\beta>0, I_{\beta}(X)=\{\xi \in \alpha: \xi=$ $\eta+\omega^{\beta}$ for some $\left.\eta<\alpha\right\}$. So, if we put $X=T\left(\omega^{\alpha}\right)$ where $\alpha$ is an infinite countable ordinal, we have that $X$ is a locally compact, $T_{2}$, scattered space such that ht $(X)=\alpha$ and $\operatorname{wd}(X)=\omega$. However, if we take $X=T\left(\omega_{1}\right)$, we have $\operatorname{ht}(X)=\operatorname{wd}(X)=\omega_{1}$. In general, if $X=T(\kappa)$ for an uncountable cardinal $\kappa$, we have $\operatorname{ht}(X)=\operatorname{wd}(X)=\kappa$.

By an LCS space, we mean a locally compact, $T_{2}$, scattered space. Suppose that $\kappa$ is an infinite cardinal and $\alpha$ is a nonzero ordinal. Let $X$ be a locally compact, $T_{2}$, scattered space. We say that $X$ is a $(\kappa, \alpha)$-LCS space, if $\operatorname{ht}(X)=\alpha$ and $\operatorname{wd}(X)=\kappa$. $X$ is $\kappa$-thin-tall, if $X$ is a $(\kappa, \alpha)$-LCS space for some ordinal $\alpha \geq \kappa^{+} . X$ is $\kappa$-thin-very tall, if $X$ is a $(\kappa, \alpha)$-LCS space for some ordinal $\alpha \geq \kappa^{++}$.

The problem of the existence of an $\left(\omega, \omega_{1}\right)$-LCS space was raised by Telgárski in 1968. After several positive solutions using additional set-theoretic hypotheses, the problem was solved in [16] by Rajagopalan, who showed that such a space exists in ZFC. A more simplified proof for this result was given by Juhász and Weiss in [8], where they even proved that there is an $(\omega, \alpha)$-LCS space for every ordinal $\alpha<\omega_{2}$.

However, it is not possible to prove in ZFC that there is an $\left(\omega, \omega_{2}\right)$-LCS space. For this, assume that we have an $\left(\omega, \omega_{2}\right)$-LCS space $X$. For every $x \in X$, consider a cone $U_{x}$ on the point $x$. Let $a_{x}$ be the subset of isolated points of $U_{x}$. Clearly, $x \neq y$ implies $a_{x} \neq a_{y}$. Hence, we can identify every point of $X$ with a subset of $I_{0}(X)$. Thus, under $\mathrm{CH}$ there is no $\left(\omega, \omega_{2}\right)$-LCS space. On the other hand, it is consistent that the continuum is large and $\left(\omega, \omega_{2}\right)$-LCS spaces do not exist (see [9] and [17]).

Nevertheless, it was shown by Baumgartner and Shelah in [1] that the existence of an $\left(\omega, \omega_{2}\right)$-LCS space is consistent with ZFC. In [15], this result was generalized, showing that it is consistent with ZFC that there is an $(\omega, \alpha)$-LCS space for every ordinal $\alpha<\omega_{3}$. It is not known whether the existence of an $\left(\omega, \omega_{3}\right)$-LCS space is consistent with ZFC.

The problem of the existence of $\left(\kappa, \kappa^{+}\right)$-LCS spaces for generic $\kappa$ was mentioned in [21]. Some consistency results on the existence of thin-tall spaces of uncountable width were shown in [10], [13] and [14]. However, it is not known whether there is in ZFC an $\left(\omega_{1}, \omega_{2}\right)$-LCS space. Also, it is unknown whether the existence of a thin-very tall space of uncountable width is consistent with ZFC.

The organization of this paper is as follows. In Section 1, we outline the construction of an $(\omega, \alpha)$-LCS space for $\alpha<\omega_{2}$ due to Juhász and Weiss. In Section 2, we 
consider some combinatorial tools introduced in [1], which are used in the forcing constructions of thin-very tall spaces. In Section 3, we describe the forcing construction of Baumgartner and Shelah. In Section 4, we outline the extension of this construction from $\omega_{2}$ to any ordinal $\alpha<\omega_{3}$. And in Section 5 , we describe some constructions of $\left(\kappa, \kappa^{+}\right)$-LCS spaces for $\kappa$ uncountable.

It is a well-known fact that the one-point compactification of a locally compact, $T_{2}$, scattered space is a Boolean space. So, the one-point compactification of a $(\kappa, \alpha)$ LCS space is a Boolean space of height $\alpha$ and width $\kappa$. On the other hand, it is known that a Boolean algebra is hereditarily atomic iff its Stone space is scattered (see [11]). So, the results we mention in this paper can be immediately transferred to the context of hereditarily atomic Boolean algebras.

For basic facts and further results and problems on scattered Boolean spaces, we refer the reader to [11] and [18].

\section{Constructions in ZCF}

For $\alpha<\omega_{2}$, we write $Z_{\alpha}=\{\alpha\} \times \omega$. The proof for the next result is similar to the one given in [8].

Theorem 1. There is an $\left(\omega, \omega_{1}\right)$-LCS space.

Proof. We construct an $\left(\omega, \omega_{1}\right)$-LCS space $X$ whose underlying set is $\bigcup\left\{Z_{\alpha}: \alpha<\right.$ $\left.\omega_{1}\right\}$. For this, we construct by transfinite induction on $\alpha<\omega_{1}$ a space $X_{\alpha}$ satisfying the following:

(1) The underlying set of $X_{\alpha}$ is $\bigcup\left\{Z_{\beta}: \beta \leq \alpha\right\}$.

(2) $X_{\alpha}$ is an $(\omega, \alpha+1)$-LCS space with $I_{\beta}\left(X_{\alpha}\right)=Z_{\beta}$ for $\beta \leq \alpha$ and $I_{\alpha+1}\left(X_{\alpha}\right)=\emptyset$.

(3) If $\beta<\alpha$ and $x \in X_{\beta}$, then a neighbourhood basis of $x$ in $X_{\beta}$ is also a neighbourhood basis of $x$ in $X_{\alpha}$.

We define $X_{0}$ as the set $Z_{0}$ with the discrete topology. Suppose $\alpha>0$. We put $Y=X_{\beta}$ if $\alpha$ is a successor ordinal $\beta+1$, and we define $Y$ as the direct union of $\left\{X_{\beta}: \beta<\alpha\right\}$ if $\alpha$ is a limit. Then, the underlying set of $X_{\alpha}$ is the union of the underlying set of $Y$ and $Z_{\alpha}$. So, our aim is to define a neighbourhood basis for each point of $Z_{\alpha}$. As $Y$ is countable infinite and non compact, $Y$ is the union of an infinite family $\mathcal{U}$ formed by pairwise disjoint compact clopen cones on points of $Y$. For this, let $\left\{x_{k}: k<\omega\right\}$ be an enumeration of $Y$. Let $U_{0}$ be a compact clopen cone on $x_{0}$. For $n>0$, let $y_{n}$ be the first element in the enumeration $\left\{x_{k}: k<\omega\right\}$ such that $y_{n} \notin U_{0} \cup \cdots \cup U_{n-1}$. We take a compact clopen cone $U_{n}$ on $y_{n}$ such that $U_{n} \cap\left(U_{0} \cup \cdots \cup U_{n-1}\right)=\emptyset$. Clearly, $\mathcal{U}=\left\{U_{n}: n<\omega\right\}$ is as required. Then, we can take a family $\left\{V_{n}: n<\omega\right\} \subseteq \mathcal{U}$ such that:

(1) If $\alpha=\beta+1$, each $V_{n}$ is a cone on a point of $I_{\beta}(Y)$. 
(2) If $\alpha$ is a limit, then for some strictly increasing sequence of ordinals $\left\langle\alpha_{n}: n<\omega\right\rangle$ cofinal in $\alpha$, we have $I_{\alpha_{n}}(Y) \cap V_{n} \neq \emptyset$ for each $n$.

Let $\left\{z_{n}: n<\omega\right\}$ be an enumeration of $Z_{\alpha}$. Consider a partition $\left\{a_{n}: n<\omega\right\}$ of $\omega$ in infinite subsets. Fix $n<\omega$. Then, we define a basic neighbourhood of $z_{n}$ as a set of the form $\left\{z_{n}\right\} \cup \bigcup\left\{V_{m}: m \in a_{n} \backslash k\right\}$ where $k<\omega$.

We define the desired space $X$ as the direct union of $\left\{X_{\alpha}: \alpha<\omega_{1}\right\}$.

Now, we outline the main construction carried out in [8].

Theorem 2. For every nonzero ordinal $\alpha<\omega_{2}$, there is an $(\omega, \alpha)$-LCS space.

Sketch of proof. When we want to extend the construction given in the proof of Theorem 1 to an ordinal $\alpha$ with $\omega_{1}<\alpha<\omega_{2}$, the problem we find is that, in general, an uncountable approximation of the space we want to construct can not be expressed as an infinite union of pairwise disjoint compact clopen cones. However, we can show by transfinite induction that for every $\alpha<\omega_{2}$ there is a Lindelöf, locally compact, $T_{2}$, scattered space $X_{\alpha}$ such that ht $\left(X_{\alpha}\right)=\alpha+1, I_{\beta}\left(X_{\alpha}\right)=Z_{\beta}$ for $\beta \leq \alpha$ and $I_{\alpha+1}\left(X_{\alpha}\right)=\emptyset$. Assume $\alpha=\omega_{1}$. Let $Y$ be the one-point compactification of the space constructed in the proof of Theorem 1. Then, we define $X_{\omega_{1}}$ as the topological sum of $\omega$ disjoint copies of $Y$.

Now, assume $\omega_{1}<\alpha<\omega_{2}$. First, suppose that $\alpha=\beta+1$ is a successor ordinal. Note that $X_{\beta}$ can not be the union of finitely many cones. Then since $X_{\beta}$ is Lindelöf, there is an infinite countable cover of $X_{\beta}$ formed by compact clopen cones on points of $X_{\beta}$. Thus, $X_{\beta}$ can be expressed as an infinite union of pairwise disjoint compact clopen cones. So, we can take a discrete family $\left\{V_{n}: n<\omega\right\}$ of compact clopen sets in $X_{\beta}$ such that each $V_{n}$ is a cone on a point of $I_{\beta}\left(X_{\beta}\right)$. Then, proceeding as in the proof of Theorem 1, we define from the family $\left\{V_{n}: n<\omega\right\}$ a neighbourhood basis for each point of $Z_{\alpha}$. Note that because of $\left\{V_{n}: n<\omega\right\}$ is discrete, the resulting space $X_{\alpha}$ is Hausdorff.

Next, suppose that $\alpha$ is a limit. Without loss of generality, we may assume that $\operatorname{cf}(\alpha)=\omega_{1}$. Let $\left\langle\alpha_{\gamma}: \gamma<\omega_{1}\right\rangle$ be a closed strictly increasing sequence of ordinals cofinal in $\alpha$. Then, by using again the Lindelöfness property, we can refine the construction given in the proof of Theorem 1 and construct by transfinite induction on $\gamma<\omega_{1}$ an approximation $Y_{\gamma}$ satisfying the following:

(1) The underlying set of $Y_{\gamma}$ is $\bigcup\left\{Z_{\beta}: \beta \leq \alpha_{\gamma}\right\}$.

(2) $Y_{\gamma}$ is a Lindelöf, locally compact, $T_{2}$, scattered space such that $I_{\beta}\left(Y_{\gamma}\right)=Z_{\beta}$ for each $\beta \leq \alpha_{\gamma}$ and $I_{\alpha_{\gamma}+1}\left(Y_{\gamma}\right)=\emptyset$.

(3) If $\beta<\gamma$ and $x \in Y_{\beta}$, then a neighbourhood basis of $x$ in $Y_{\beta}$ is also a neighbourhood basis of $x$ in $Y_{\gamma}$.

Let $Y$ be the one-point compactification of the direct union of $\left\{Y_{\gamma}: \gamma<\omega_{1}\right\}$. Let $X_{\alpha}$ be the topological sum of $\omega$ disjoint copies of $Y$. Then, $X_{\alpha}$ is as required. 
By means of a refinement of the construction given in the proof of Theorem 1, Dow and Simon proved in [4] that there are $2^{\omega_{1}}$ (as many as possible) pairwise nonhomeomorphic $\left(\omega, \omega_{1}\right)$-LCS spaces. The proof of this result can be immediately generalized to any ordinal $\alpha$ with $\omega_{1} \leq \alpha<\omega_{2}$. Briefly, the idea of the proof is as follows. We fix an ordinal $\alpha$ with $\omega_{1} \leq \alpha<\omega_{2}$. The underlying set of the spaces we want to construct is $\alpha \times \omega$. For every $n<\omega$, we put $C_{n}=\omega_{1} \times\{n\}$. Suppose that $X$ is an LCS space of underlying set $\alpha \times \omega$ such that $I_{\xi}(X)=\{\xi\} \times \omega$ for any $\xi<\alpha$ and $C_{n}$ is closed in the subspace $X \backslash X^{\omega_{1}}$ for any $n<\omega$. If $S$ is an stationary subset of $\omega_{1}$, we say that $S$ is associated to $X$ if for every $n<\omega$ and every limit ordinal $\xi<\omega_{1}$, if $\xi \in S$ then for every neighbourhood $U$ of $(\xi, n)$ there is a $\zeta<\xi$ such that $\{(\mu, n): \zeta<\mu \leq \xi\} \subseteq U$, and if $\xi \notin S$ then there is a neighbourhood $U$ of $(\xi, n)$ such that $U \cap C_{n}=\{(\xi, n)\}$. By refining the constructions carried out above, we can show that every stationary subset of $\omega_{1}$ is associated to some LCS space. And it can be checked that if $S, S^{\prime}$ are stationary subsets of $\omega_{1}$ with $\left|S \backslash S^{\prime}\right|=\omega_{1}$ and $X, Y$ are LCS spaces such that $S$ is associated to $X$ and $S^{\prime}$ is associated to $Y$, then $X$ and $Y$ are not homeomorphic. Let $\left\{S_{\xi}: \xi<2^{\omega_{1}}\right\}$ be a collection of stationary subsets of $\omega_{1}$ such that if $\mu<\xi<2^{\omega_{1}}$, then $S_{\xi} \backslash S_{\mu}$ is stationary. Consider a collection $\left\{X_{\xi}: \xi<2^{\omega_{1}}\right\}$ of LCS spaces such that $S_{\xi}$ is associated to $X_{\xi}$ for $\xi<2^{\omega_{1}}$. If $\mu<\xi<2^{\omega_{1}}$, we deduce that $X_{\mu}$ and $X_{\xi}$ are not homeomorphic. So, we have the following result.

Theorem 3. For every ordinal $\alpha$ with $\omega_{1} \leq \alpha<\omega_{2}$, there are $2^{\omega_{1}}$ pairwise nonhomeomorphic $(\omega, \alpha)$-LCS spaces.

It was also proved in [4] that for every countable group $G$ there exist $2^{\omega_{1}}$ pairwise non-homeomorphic $\left(\omega, \omega_{1}\right)$-LCS spaces for which $G$ is the non-trivial autohomeomorphism group.

\section{LCS structures}

The following definition is an immediate generalization of the notion of partial order introduced in [1].

Let $\gamma$ be a nonzero ordinal. Suppose that $T=\bigcup\left\{T_{\alpha}: \alpha<\gamma\right\}$ where each $T_{\alpha}$ is a nonempty set and $T_{\alpha} \cap T_{\beta}=\emptyset$ for $\alpha<\beta<\gamma$. If $s \in T_{\alpha}$ for $\alpha<\gamma$, we write $\pi(s)=\alpha$. Suppose that $\leq$ is a partial ordering on $T$. Assume that $i:[T]^{2} \rightarrow[T]^{<\omega}$. Then, we say that $(T, \leq, i)$ is an LCS structure (on $T$ ) if the following conditions are satisfied:

$(/)(1)$ If $s<t$, then $\pi(s)<\pi(t)$.

(2) If $\alpha<\beta$ and $t \in T_{\beta}$, then $\left\{s \in T_{\alpha}: s<t\right\}$ is infinite.

(3) For all $\{s, t\} \in[T]^{2}$ the following holds:

(a) If $s<t$, then $i\{s, t\}=\{s\}$.

(b) For all $v \in i\{s, t\}, v \leq s, t$. 
(c) For every $u \leq s, t$ there is a $v \in i\{s, t\}$ with $u \leq v$.

Note that, intuitively, $\pi(s)$ denotes the level of $s$ in $T$, and $i\{s, t\}$ is an analogue of the notion of infimum of $s, t$, if $i\{s, t\}$ is nonempty.

The following result, which will be used without explicit mention, permits us to construct LCS spaces from LCS structures in a natural way.

Proposition 1. Suppose that $(T, \leq, i)$ is an LCS structure with $T=\bigcup\left\{T_{\alpha}: \alpha<\gamma\right\}$. Then, we can define a topology $\sigma$ on $T$ such that $(T, \sigma)$ is a locally compact, $T_{2}$, scattered space with $I_{\alpha}((T, \sigma))=T_{\alpha}$ for each $\alpha<\gamma$.

Proof. For every $t \in T$, let $C(t)=\{s \in T: s \leq t\}$. We infer from $(/)(3)$ that if $s, t$ are different elements of $T$, then $C(s) \cap C(t)=\bigcup\{C(u): u \in i\{s, t\}\}$. Let

$$
B=\left\{C(t) \backslash\left(C\left(t_{1}\right) \cup \cdots \cup C\left(t_{n}\right)\right): n<\omega, t_{1}, \ldots, t_{n}<t\right\} .
$$

We have that $B$ is a clopen base for a Hausdorff topology $\sigma$ on $T$. Clearly, $C(t)$ is compact for every $t \in T$. And we deduce from $(/)(1)-(2)$ that $(T, \sigma)$ is a scattered space such that $I_{\alpha}((T, \sigma))=T_{\alpha}$ for each $\alpha<\gamma$.

It is not known whether every LCS space can be constructed from an LCS structure.

On the other hand, a class of LCS structures satisfying additional properties, the so-called PCF structures, have interest in the theory of cardinal arithmetic, concerning Shelah's result that if $\omega_{\omega}$ is a strong limit cardinal, then $2^{\omega_{\omega}}<\omega_{\omega_{4}}$ (see [20]). Suppose that $(T, \preceq, i)$ is an LCS structure. Let $\sigma$ be the topology associated to $(T, \preceq)$ in Proposition 1. If $S \subseteq T$, we denote by $\bar{S}$ the closure of $S$ in $(T, \sigma)$. Then we say that $(T, \preceq, i)$ is a PCF structure, if the following conditions hold:

(1) $T$ is an infinite successor ordinal.

(2) For every $\mu, \xi \in T, \mu \prec \xi$ implies $\mu \in \xi$.

(3) $\bar{\omega}=T$.

(4) If $I \subseteq T$ is an interval, then $\bar{I}$ is also an interval.

(5) If $\mu$ is an ordinal of uncountable cofinality contained in $T$, then there is a closed unbounded set $C_{\mu} \subseteq \mu$ such that $\overline{C_{\mu}} \subseteq \mu+1$.

(6) $(T, \sigma)$ is compact.

By a result of Foreman and Magidor, it is known that if $(T, \preceq, i)$ is a PCF structure with $T=\bigcup\left\{T_{\alpha}: \alpha<\gamma\right\}$, then $\left|T_{\alpha}\right| \leq|\alpha+\omega|$ for each $\alpha<\gamma$ (see [19]).

The interest of the notion of a PCF structure lies in the fact that for the proof of Shelah's theorem stated above, it is shown by means of a combinatorial argument that no PCF structure of size $\geq \omega_{4}$ exists. Then, the non existence of PCF structures of size $\omega_{3}$ would improve Shelah's bound, and so we would have $2^{\omega_{\omega}}<\omega_{\omega_{3}}$ provided 
$\omega_{\omega}$ is a strong limit cardinal. However, it is not known whether the non existence of PCF structures of size $\omega_{3}$ can be proved in ZFC. Nevertheless, it was shown in [19] that the existence of a PCF structure of size $\omega_{2}$ is consistent with ZFC, and so we can not hope to improve the bound on $2^{\omega_{\omega}}$ to $\omega_{\omega_{2}}$, at least by using the argument given in the proof of Shelah's theorem.

In what follows, we shall construct LCS spaces from LCS structures. Then, in order to construct by forcing thin-very tall spaces of countable width, we consider an ordinal $\gamma$ with $\omega_{2} \leq \gamma<\omega_{3}$ and we put $T=\bigcup\left\{T_{\alpha}: \alpha<\gamma\right\}$ where each $T_{\alpha}=\{\alpha\} \times \omega$. We can define a natural notion of forcing $\mathbb{P}_{\gamma}$ for adding a partial ordering $\leq$ on $T$ and a function $i:[T]^{2} \rightarrow[T]^{<\omega}$ such that $(T, \leq, i)$ is an LCS structure. For this, let $P_{\gamma}$ be the set of all $p=\left(x_{p}, \leq_{p}, i_{p}\right)$ such that the following holds:

(*)(1) $x_{p}$ is a finite subset of $T$.

$(2) \leq_{p}$ is a partial ordering on $x_{p}$ such that for every $s, t \in x_{p}, s<_{p} t$ implies $\pi(s)<\pi(t)$.

(3) $i_{p}:\left[x_{p}\right]^{2} \rightarrow\left[x_{p}\right]^{<\omega}$ such that for all $\{s, t\} \in\left[x_{p}\right]^{2}$ the following holds:

(a) If $s<_{p} t$, then $i_{p}\{s, t\}=\{s\}$.

(b) For all $v \in i_{p}\{s, t\}, v \leq_{p} s, t$.

(c) For every $u \leq_{p} s, t$ there is a $v \in i_{p}\{s, t\}$ such that $u \leq_{p} v$.

If $p, q \in P_{\gamma}$, we put $p \leq_{\gamma} q$ iff $x_{p} \supseteq x_{q}, \leq_{p} \uparrow x_{q}=\leq_{q}$ and $i_{p} \uparrow\left[x_{q}\right]^{2}=i_{q}$.

We put $\mathbb{P}_{\gamma}=\left(P_{\gamma}, \leq_{\gamma}\right)$.

Proposition 2. $\mathbb{P}_{\gamma}$ adjoins an LCS structure on $T$.

Proof. Suppose that $G$ is $\mathbb{P}_{\gamma}$-generic. Clearly, for each $t \in T,\left\{p \in P_{\gamma}: t \in x_{p}\right\}$ is dense in $\mathbb{P}_{\gamma}$. So, $T=\bigcup\left\{x_{p}: p \in G\right\}$. Now, let $\leq=\bigcup\left\{\leq_{p}: p \in G\right\}$ and $i=\bigcup\left\{i_{p}\right.$ : $p \in G\}$. We immediately obtain condition $(/)(1)$ from $(*)(2)$, and $(/)(3)$ from $(*)(3)$. Also, by using $(*)(1)$, it is easy to check that for $\alpha<\beta, t \in T_{\beta}$ and $n<\omega$, the set $\left\{p \in P_{\gamma}\right.$ : there is an $s=(\alpha, m)$ for some $m>n$ with $\left.s<_{p} t\right\}$ is dense in $\mathbb{P}_{\gamma}$. So, condition $(/)(2)$ holds too.

However, we shall need to use a special function $F:[\gamma]^{2} \rightarrow[\gamma] \leq \omega$ in order to refine the forcing $\mathbb{P}_{\gamma}$ and obtain a notion of forcing that preserves cardinals.

If $p=\left(x_{p}, \leq_{p}, i_{p}\right) \in P_{\gamma}$ and $s, t \in x_{p}$, we say that $s, t$ are comparable in $p$, if either $s \leq_{p} t$ or $t \leq_{p} s$.

Let $F:[\gamma]^{2} \rightarrow[\gamma] \leq \omega$. We define $P_{\gamma}^{F}$ as the set of all $p \in P_{\gamma}$ such that the following additional condition holds:

$(*)(4)$ If $s, t \in x_{p}$ are not comparable in $p$, then $\pi\left[i_{p}\{s, t\}\right] \subseteq F\{\pi(s), \pi(t)\}$. 
We define the partial ordering $\leq$ on $P_{\gamma}^{F}$ in the same way we defined the partial ordering on $P_{\gamma}$. We put $\mathbb{P}_{\gamma}^{F}=\left(P_{\gamma}^{F}, \leq\right)$.

Then, we need to strengthen the partial order $\mathbb{P}_{\gamma}^{F}$ in order to obtain the desired cardinal-preserving notion of forcing. For this, we introduce the following combinatorial notion.

Let $Z$ be a set of ordinals of order type $\omega_{2}$. Let $F:[Z]^{2} \rightarrow[Z]^{\leq \omega}$ such that $F\{\alpha, \beta\} \subseteq \min \{\alpha, \beta\}$ for every $\{\alpha, \beta\} \in[Z]^{2}$. Let $A$ be an uncountable set of finite subsets of $Z$. We say that $A$ is admissible for $F$, if the following condition holds:

(\#) For every $a, b \in A$ with $a \neq b, \alpha \in a \backslash b, \beta \in b \backslash a$ and $\tau \in a \cap b$ we have:

(1) $\tau<\alpha, \beta$ implies $\tau \in F\{\alpha, \beta\}$.

(2) $\tau<\beta$ implies $F\{\alpha, \tau\} \subseteq F\{\alpha, \beta\}$.

Then, we say that $F$ is a $\Delta$-function on $Z$, if any uncountable set of finite subsets of $Z$ contains an admissible subset for $F$.

A weaker form of the notion of a $\Delta$-function was used in [1] to prove the consistency of the existence of an $\left(\omega, \omega_{2}\right)$-LCS space. In [1], Baumgartner and Shelah obtained the weaker $\Delta$-function by forcing. However, by using Todorčević's $\rho$ function, Veličković proved the following result (see [2]).

Proposition 3. $\square_{\omega_{1}}$ implies the existence of a $\Delta$-function on $\omega_{2}$.

Clearly, a $\Delta$-function on $\omega_{2}$ can be transferred to a $\Delta$-function on any set of ordinals of order type $\omega_{2}$.

Then, by using this combinatorial notion, we can define a c.c.c. partial order $\mathbb{Q}_{\gamma}$ that adjoins an $(\omega, \gamma)$-LCS space. In order to show that $\mathbb{Q}_{\gamma}$ is c.c.c., we can find in any uncountable set $D$ of forcing conditions in $\mathbb{Q}_{\gamma}$ an uncountable subset $E \subseteq D$ such that $\left\{x_{p}: p \in E\right\}$ is a $\Delta$-system and any pair of conditions in $E$ are isomorphic by means of a bijection that fixes the elements of the root of the $\Delta$-system. So, we shall need the following two definitions.

For $p, q \in P_{\gamma}$, suppose that $f: x_{p} \rightarrow x_{q}$ is a bijection. We say that $f$ is adequate, if the following holds:

(1) For every $s, t \in x_{p}, \pi(s)<\pi(t)$ iff $\pi(f(s))<\pi(f(t))$.

(2) For all $s=(\alpha, n) \in x_{p}, f(\alpha, n)=(\beta, m)$ implies $n=m$.

We say that an uncountable set $E \subseteq P_{\gamma}$ is separated, if the following conditions are satisfied:

(1) $\left\{x_{p}: p \in E\right\}$ forms a $\Delta$-system with root $x$. 
(2) For each $\alpha<\gamma$, either $x_{p} \cap T_{\alpha}=x \cap T_{\alpha}$ for every $p \in E$, or there is at most one $p \in E$ such that $x_{p} \cap T_{\alpha} \neq \emptyset$.

(3) For every $p, q \in E$ there is an adequate bijection $h_{p q}: x_{p} \rightarrow x_{q}$ such that:

(a) For any $s \in x, h_{p q}(s)=s$.

(b) For all $s, t \in x_{p}, s \leq_{p} t$ iff $h_{p q}(s) \leq_{q} h_{p q}(t)$.

(c) For all $\{s, t\} \in\left[x_{p}\right]^{2}, h_{p q}\left[i_{p}\{s, t\}\right]=i_{q}\left\{h_{p q}(s), h_{p q}(t)\right\}$.

By means of a standard combinatorial argument, we can show that every uncountable subset of $P_{\gamma}$ contains a separated subset.

\section{Baumgartner-Shelah's theorem}

In this section, we show the following result.

Theorem 4. Con $(Z F C) \rightarrow$ Con $\left(Z F C+\right.$ "there is an $\left(\omega, \omega_{2}\right)-L C S$ space").

Assume that $\square_{\omega_{1}}$ holds. Let $F$ be a $\Delta$-function on $\omega_{2}$. Then, we define $\mathbb{Q}=\mathbb{P}_{\omega_{2}}^{F}$.

Proposition 4. $\mathbb{Q}$ is c.c.c.

Proof. Let $D$ be an uncountable subset of forcing conditions in $\mathbb{Q}$. By thinning out $D$ if necessary, we may assume that $D$ is separated. We denote by $x$ the root of the $\Delta$-system $\left\{x_{p}: p \in D\right\}$. Since $D$ is separated and each $F\{\alpha, \beta\}$ is countable, we infer by $(*)(4)$ that $i_{p} \uparrow[x]^{2}=i_{q} \uparrow[x]^{2}$ for all $p, q \in D$.

We put $a_{p}=\pi\left[x_{p}\right]$ for $p \in D$, and $a=\pi[x]$. By the definition of a $\Delta$-function, there is an uncountable subset $E$ of $D$ such that $\left\{a_{p}: p \in E\right\}$ is admissible for the function $F$. Now, we can show that $E$ is linked. For this, suppose that $p, q$ are different elements of $E$. In order to show that $p, q$ are compatible in $\mathbb{Q}$, we define the condition $r=r(p, q, F)=\left(x_{r}, \leq_{r}, i_{r}\right)$ as follows. We put $x_{r}=x_{p} \cup x_{q}$. If $s, t \in x_{r}$, we define $s \leq_{r} t$ iff $s \leq_{p} t$ or $s \leq_{q} t$ or there is a $u \in x$ such that either $s<_{p} u<_{q} t$ or $s<_{q} u<_{p} t$. Now we define $i_{r}$ by: $i_{r} \uparrow\left[x_{p}\right]^{2}=i_{p}, i_{r} \uparrow\left[x_{q}\right]^{2}=i_{q}$; and if $s \in x_{p} \backslash x_{q}$ and $t \in x_{q} \backslash x_{p}$, then $i_{r}\{s, t\}=\left\{u \in x_{r}: u<_{r} s, t\right.$ and $\left.\pi(u) \in F\{\pi(s), \pi(t)\}\right\}$ if $s, t$ are not comparable in $r, i_{r}\{s, t\}=\{s\}$ if $s<_{r} t$ and $i_{r}\{s, t\}=\{t\}$ if $t<_{r} s$.

To show that $r \in Q$, it is immediate to check all the conditions in the definition of $Q$ except condition $(*)(3)(c)$. It is also easy to verify $(*)(3)(c)$, if $s, t \in x_{p}$ or $s, t \in x_{q}$. So, assume that $s \in x_{p} \backslash x_{q}$ and $t \in x_{q} \backslash x_{p}$. Without loss of generality, we may assume that $s, t$ are not comparable in $r$. So, suppose $u<_{r} s, t$. Our aim is to find a $v \in x_{r}$ such that $u \leq_{r} v<_{r} s, t$ and $\pi(v) \in F\{\pi(s), \pi(t)\}$. Suppose that $u \in x_{p}$ (the case $u \in x_{q}$ is parallel). As $u<_{r} t$, there is a $w \in x$ such that $u \leq_{p} w<_{q} t$. Since $s, t$ are not comparable in $r$, we have $s \mathbb{Z}_{p} w$. Assume $w<_{p} s$. We obtain from $(\#)(1)$ that $\pi(w) \in F\{\pi(s), \pi(t)\}$. Thus, since we have $u \leq_{p} w<_{r} s, t$, we are done. Now, assume that $w \nless_{p} s$. Let $v \in i_{p}\{s, w\}$ such that $u \leq_{p} v$. We infer from $(*)(4)$ and 
(\#)(2) that $\pi(v) \in F\{\pi(s), \pi(w)\} \subseteq F\{\pi(s), \pi(t)\}$. Thus, as we have $u \leq_{p} v<_{r} s, t$, we are done.

Now, proceeding as in Proposition 2, we infer that $\mathbb{Q}$ adjoins an $\left(\omega, \omega_{2}\right)$-LCS space.

\section{Constructions of height greater than $\omega_{2}$}

Suppose that $M$ is a model of ZFC in which there exists an $\left(\omega, \omega_{2}\right)$-LCS space. Note that the argument given in the proof of Theorem 2 can not be carried out to construct in $M$ an $(\omega, \alpha)$-LCS space for every $\alpha<\omega_{3}$. For example, for $\alpha=\omega_{2} \cdot \omega_{2}$ we would need $\omega_{2}$ approximations of the required space, but then we can not assume that the $\beta$-approximations of the space are Lindelöf for $\beta \geq \omega_{1}$.

Then, our purpose in this section is to describe a forcing for adding an $(\omega, \alpha)$-LCS space for any $\alpha<\omega_{3}$.

By an ordinal interval we mean a half-open interval $[\alpha, \beta)$ where $\alpha, \beta$ are ordinals with $\alpha<\beta$. If $I=[\alpha, \beta)$ is an ordinal interval, we write $I^{-}=\alpha$ and $I^{+}=\beta$.

To develop our forcing construction we need to introduce the combinatorial notion of a tree of intervals. Basically, the idea for this notion is as follows. For an ordinal $\gamma$ with $\omega_{2}<\gamma<\omega_{3}$, we have a collection of ordinal intervals such that each interval in the collection has a (finite) level. We define $[0, \gamma)$ as the only interval at level 0 . If $I$ is an interval at level $n$ with more than one element, we take a partition $\left\{I_{\alpha}: \alpha<\lambda\right\}$ of $I$ in ordinal intervals with $I_{\alpha}<I_{\beta}$ for $\alpha<\beta<\lambda$ such that $\lambda$ is finite if $I^{+}$is a successor ordinal and $\lambda=\operatorname{cf}\left(I^{+}\right)$if $I^{+}$is a limit. Now, we consider that the intervals $I_{\alpha}$ are at level $n+1$. So, we get the following notion.

Let $\gamma$ be a nonzero ordinal. A tree of intervals on $\gamma$ is a collection $\mathcal{I}=\bigcup_{n<\omega} \mathcal{I}_{n}$ of ordinal intervals such that:

(1) $\mathcal{I}_{0}=\{[0, \gamma)\}$.

(2) $\mathcal{I}_{n}$ is a partition of $[0, \gamma)$ for each $n<\omega$.

(3) $\mathcal{I}_{n+1}$ is a refinement of $\mathcal{I}_{n}$ for each $n<\omega$.

(4) For every $I \in \mathcal{I}_{n}$, if $I^{+}$is a limit ordinal then $\left\{J^{-}: J \in \mathcal{I}_{n+1}, J \subseteq I\right\}$ is a sequence of order type $\operatorname{cf}\left(I^{+}\right)$, and otherwise $\left\{J \in \mathcal{I}_{n+1}: J \subseteq I\right\}$ is finite.

(5) $\{\alpha\} \in \mathcal{I}$ for every $\alpha<\gamma$.

We give an example. For $\gamma=\omega_{2} \cdot \omega_{2}$, we define the tree of intervals $\mathcal{I}=\bigcup_{n<\omega} \mathcal{I}_{n}$ on $\gamma$ by $\mathcal{I}_{0}=\left\{\left[0, \omega_{2} \cdot \omega_{2}\right)\right\}, \mathcal{I}_{1}=\left\{\left[\omega_{2} \cdot \xi, \omega_{2} \cdot(\xi+1)\right): \xi<\omega_{2}\right\}$ and $\mathcal{I}_{n}=\{\{\xi\}: \xi<$ $\left.\omega_{2} \cdot \omega_{2}\right\}$ for $n \geq 2$.

Note that if $\mathcal{I}$ is a tree of intervals, we have that for every $I, J \in \mathcal{I}, I \subseteq J$ or $J \subseteq I$ or $I \cap J=\emptyset$.

The following result is easy to prove. 
Proposition 5. For every nonzero ordinal $\gamma$, there is a tree of intervals on $\gamma$.

Suppose that $\mathcal{I}=\bigcup_{n<\omega} \mathcal{I}_{n}$ is a tree of intervals on an ordinal $\gamma$. If $\alpha<\gamma$ and $n<\omega$, we denote by $I(\alpha, n)$ the interval of $\mathcal{I}_{n}$ that contains $\alpha$. Then, suppose that $\alpha<\beta<\gamma$ and $n<\omega$. We say that $n$ is the level where $\alpha, \beta$ separate (in $\mathcal{I}$ ) if $I(\alpha, n)=I(\beta, n)$ but $I(\alpha, n+1) \neq I(\beta, n+1)$. Then, we write $j(\alpha, \beta)=n$. Clearly, any pair of different elements of $\gamma$ separate at some level.

Assume that $\square_{\omega_{1}}$ holds. We fix an ordinal $\gamma$ such that $\omega_{2}<\gamma<\omega_{3}$ and a tree of intervals $\mathcal{I}=\bigcup_{n<\omega} \mathcal{I}_{n}$ on $\gamma$. First, we define the special function we use in our forcing. If $I \in \mathcal{I}_{n}$, we put $Z_{I}=\left\{J^{-}: J \in \mathcal{I}_{n+1}, J \subseteq I\right\}$, and then we choose a function $G_{I}:\left[Z_{I}\right]^{2} \rightarrow\left[Z_{I}\right]^{\leq \omega}$ as follows: if $\operatorname{cf}\left(I^{+}\right)<\omega_{2}$, we define $G_{I}\{\alpha, \beta\}=Z_{I} \cap \alpha$ for $\alpha, \beta \in Z_{I}$ with $\alpha<\beta$; and if $\operatorname{cf}\left(I^{+}\right)=\omega_{2}$, we choose $G_{I}$ as a $\Delta$-function on $Z_{I}$. Then, we define the function $G:[\gamma]^{2} \rightarrow[\gamma] \leq \omega$ as follows. Suppose $\alpha<\beta<\gamma$. Let $n=j(\alpha, \beta)$. Let $I=I(\alpha, n)=I(\beta, n), J=I(\alpha, n+1), K=I(\beta, n+1)$. Then, we define $G\{\alpha, \beta\}=G_{I}\left\{J^{-}, K^{-}\right\} \cup\left\{I^{-}\right\}$.

For each $\alpha<\gamma$ we denote by $l(\alpha)$ the least $n$ such that there is an $I \in \mathcal{I}_{n}$ with $I^{-}=\alpha$. Note that by condition (5) in the definition of a tree of intervals, this natural number exists. Clearly, if $I, J \in \mathcal{I}$ are such that $J \subseteq I$ and $J^{+}<I^{+}$, then $l\left(J^{+}\right)>l\left(I^{+}\right)$.

Consider $\alpha, \beta$ with $\alpha<\beta<\gamma$. Let $n=j(\alpha, \beta)$ and $J=I(\alpha, n+1)$. For $k<\omega$, we write $\beta_{k}=I(\beta, k)^{-}$. Then, we define the walk from $\alpha$ to $\beta$ via $\mathcal{I}$ by $w(\alpha, \beta)=$ $\left\langle\alpha, \beta_{n+1}, \beta_{n+2}, \ldots, \beta_{l(\beta)-1}, \beta\right\rangle$ if $\alpha=J^{-}$, and by $w(\alpha, \beta)=\left\langle\alpha, J^{+}, \beta_{n+1}, \beta_{n+2}, \ldots\right.$, $\left.\beta_{l(\beta)-1}, \beta\right\rangle$ if $\alpha \neq J^{-}$. This notion resembles the notion of a walk considered by Todorčević in [22].

Now, we introduce the required forcing. We define $Q_{\gamma}$ as the set of all $p=\left(x_{p}, \leq_{p}\right.$, $\left.i_{p}\right) \in P_{\gamma}^{G}$ such that the following two additional conditions hold:

$(*)(5)$ If $s, t$ are different elements of $x_{p}$ with $\pi(s)=\pi(t)$, then $i_{p}\{s, t\}=\emptyset$.

(6) If $s<_{p} t$, there are $u_{1}, \ldots, u_{n} \in x_{p}$ with $s<_{p} u_{1} \leq_{p} \ldots \leq_{p} u_{n} \leq_{p} t$ such that $w(\pi(s), \pi(t))=\left\langle\pi(s), \pi\left(u_{1}\right), \ldots, \pi\left(u_{n}\right), \pi(t)\right\rangle$.

We define the partial ordering $\leq$ on $Q_{\gamma}$ in the same way we defined the partial ordering on $P_{\gamma}$. We put $\mathbb{Q}_{\gamma}=\left(Q_{\gamma}, \leq\right)$.

Proposition 6. $\mathbb{Q}_{\gamma}$ is c.c.c.

Sketch of proof. Let $D$ be an uncountable subset of $Q_{\gamma}$. Without loss of generality, we may assume that $D$ is separated. We denote by $x$ the root of the $\Delta$-system $\left\{x_{p}: p \in D\right\}$. We write $a_{p}=\pi\left[x_{p}\right]$ for $p \in D$, and $a=\pi[x]$. Note that since $D$ is separated and $G:[\gamma]^{2} \rightarrow[\gamma] \leq \omega$, we have $i_{p} \uparrow[x]^{2}=i_{q} \uparrow[x]^{2}$ for all $p, q \in D$. Also, by means of a combinatorial argument, it is not difficult to show that there is an uncountable set $E \subseteq D$ such that: 
(i) For all $p, q \in E$, if $s \in x_{p}$ and $s^{\prime}=h_{p q}(s)$, we have $l(\pi(s))=l\left(\pi\left(s^{\prime}\right)\right)$.

(ii) For every $I \in \mathcal{I}_{n}$ with $\left|Z_{I}\right|=\omega_{2}$, if $\left\{a_{p} \cap Z_{I}: p \in E\right\}$ is uncountable, then $\left\{a_{p} \cap Z_{I}: p \in E\right\}$ is admissible for the function $G_{I}$.

Suppose that $p, q$ are different elements of $E$. To verify that $p, q$ are compatible in $\mathbb{Q}_{\gamma}$, we define the condition $r=r(p, q, G)=\left(x_{r}, \leq_{r}, i_{r}\right)$ as in the proof of Proposition 4 replacing the function $F$ by the function $G$. Then, to show that $r \in Q_{\gamma}$ the crucial step is the verification of condition $(*)(3)(c)$. For this, we consider the non trivial case in which $s \in x_{p} \backslash x_{q}, t \in x_{q} \backslash x_{p}$ and $s, t$ are not comparable in $r$. We may assume $\pi(s)<\pi(t)$. Suppose that $u<_{r} s, t$. We deal with the case $u \in x_{p}$. The considerations in the case $u \in x_{q}$ are analogous. Then, we need to show:

$(+)$ There is a $v \in x_{r}$ such that $u \leq_{r} v<_{r} s, t$ and $\pi(v) \in G\{\pi(s), \pi(t)\}$.

Let $n=j(\pi(s), \pi(t))$. Put $I=I(\pi(s), n)=I(\pi(t), n), J=I(\pi(s), n+1)$ and $K=I(\pi(t), n+1)$. First, assume that $\pi(u) \notin I$. Then, we can show that there is a $v \in x_{r}$ with $u<_{r} v<_{r} s, t$ and $\pi(v)=I^{-}$, and thus since $I^{-} \in G\{\pi(s), \pi(t)\}$, we are done. For this, first we infer from $u<_{r} t$ that there is a $w \in x$ such that $u \leq_{p} w<_{q} t$. Suppose that $\pi(w) \notin I$. If $\pi(w) \in I$, the considerations are analogous. Note that $I^{-}$is in the walk from $\pi(u)$ to $\pi(s)$ and also in the walk from $\pi(w)$ to $\pi(t)$. Thus since $u<_{p} s$ and $w<_{q} t$, we infer from $(*)(6)$ that there are $v_{1} \in x_{p}$ and $v_{2} \in x_{q}$ with $\pi\left(v_{1}\right)=\pi\left(v_{2}\right)=I^{-}$such that $u<_{p} v_{1} \leq_{p} s$ and $w<_{q} v_{2}<_{q} t$. Thus $I^{-} \in a$, and therefore $v_{1}, v_{2} \in x$. Then as $u<_{p} v_{1}, v_{2}$, we infer from $(*)(3)(c)$ and $(*)(5)$ that $v_{1}=v_{2}$, and so we are done.

Now, suppose that $\pi(u) \in I$. As $I^{-} \in G\{\pi(s), \pi(t)\}$, we may assume without loss of generality that $\pi(u) \neq I^{-}$. Then, we can prove that $\pi(u) \notin J$. For this, assume on the contrary that $\pi(u) \in J$. As $u<_{q} t$, there is a $w \in x$ with $u \leq_{p} w<_{q} t$. Assume that $w \in J$ (otherwise, the argument is easier). Let $t^{\prime}=h_{q p}(t)$. Since $w<_{q} t$ and $w \in x$, we infer that $w<_{p} t^{\prime}$. Note that $J^{+}$is in the walk from $\pi(w)$ to $\pi(t)$. Then, we deduce from (i) and $(*)(6)$ that $J^{+}$is also in the walk from $\pi(w)$ to $\pi\left(t^{\prime}\right)$, and hence $\pi\left(t^{\prime}\right) \in I \backslash J$. So, $G\left\{\pi(s), \pi\left(t^{\prime}\right)\right\} \subseteq J^{-}$if $I^{-} \neq J^{-}$, and $G\left\{\pi(s), \pi\left(t^{\prime}\right)\right\}=\left\{J^{-}\right\}$ if $I^{-}=J^{-}$. Now as $\pi(u) \in J, \pi(u) \neq I^{-}$and $u<_{p} s, t^{\prime}$, it is easy to deduce that $s, t^{\prime}$ are comparable in $p$ by using conditions $(*)(3)(c)$ and $(*)(4)$. Then since $\pi(s)<\pi\left(t^{\prime}\right)$, we have $s<_{p} t^{\prime}$. By $(*)(6)$, we find a $v_{1} \in x_{p} \cap T_{J^{+}}$such that $s<_{p} v_{1} \leq_{p} t^{\prime}$. Also, as $w<_{q} t$, we deduce from $(*)(6)$ that there is a $v_{2} \in x_{q} \cap T_{J}$ such that $w<_{q} v_{2} \leq_{q} t$. Thus $J^{+} \in a$, and hence $v_{1}, v_{2} \in x$. Now since $u<_{p} v_{1}, v_{2}$, we infer from $(*)(3)(c)$ and $(*)(5)$ that $v_{1}=v_{2}$, therefore $s<_{p} v_{1}<_{q} t$, and hence $s<_{r} t$, which contradicts the assumption that $s, t$ are incomparable in $r$. So, $\pi(u) \notin J$.

Then, by using again condition $(*)(6)$, we find elements $v_{1} \in x_{p} \cap T_{J^{-}}$and $v_{2} \in$ $x_{r} \cap T_{K^{-}}$with $u<_{p} v_{1} \leq_{p} s$ and $u<_{r} v_{2} \leq_{r} t$. Note that as $\pi(s)<\pi(t)$, we have $\pi\left(v_{1}\right)<\pi\left(v_{2}\right)$. As $\pi\left(v_{1}\right) \in J$, by the argument given in the preceding paragraph, we infer that $v_{1}, v_{2}$ are incomparable in $r$. Also, by using $(*)(6)$, we may assume that $\pi(u) \in Z_{I}$. So, $\pi(u), \pi\left(v_{1}\right), \pi\left(v_{2}\right) \in Z_{I}$. To show condition $(+)$, we consider the case in which $v_{1} \in x_{p} \backslash x_{q}$ and $v_{2} \in x_{q} \backslash x_{p}$ (otherwise, the situation is easier to 
handle). Without loss of generality, we may suppose that $\left|Z_{I}\right|=\omega_{2}$. Since $u<_{r} v_{2}$, there is a $w \in x$ such that $u \leq_{p} w<_{q} v_{2}$. As $w<_{q} v_{2}$ and $w \in x$, we have $w<_{q^{\prime}} h_{q q^{\prime}}\left(v_{2}\right)$ for every $q^{\prime} \in E$. Thus since $\pi\left(v_{2}\right) \in Z_{I}$, we infer from (i) and $(*)(6)$ that $\left\{a_{p} \cap Z_{I}: p \in E\right\}$ is uncountable. Now, by using condition (ii), we can proceed as in the proof of Proposition 4 (replacing $\omega_{2}$ by $Z_{I}$ ), and then we obtain a $v \in x_{r} \cap Z_{I}$ such that $u \leq_{r} v<_{r} v_{1}, v_{2}$ and $\pi(v) \in G_{I}\left\{\pi\left(v_{1}\right), \pi\left(v_{2}\right)\right\}$. But since $G_{I}\left\{\pi\left(v_{1}\right), \pi\left(v_{2}\right)\right\} \subseteq G\{\pi(s), \pi(t)\}$, we are done.

Then, we can adapt to $\mathbb{Q}_{\gamma}$ the argument for the proof of Proposition 2 and show that $\mathbb{Q}_{\gamma}$ adjoins an $(\omega, \gamma)$-LCS space. Thus, since the countable chain condition is preserved by finite support iterated forcing constructions, we obtain the following result.

Theorem 5. Con $(Z F C) \rightarrow$ Con $\left(Z F C+\right.$ "for every $\alpha<\omega_{3}$ there is an $(\omega, \alpha)-L C S$ space").

In fact, it is proved that $M A+2^{\omega}>\omega_{2}+\square_{\omega_{1}}$ implies the existence of an $(\omega, \alpha)$-LCS space for every $\alpha<\omega_{3}$.

\section{Constructions of uncountable width}

The situation is more difficult when we want to construct thin-tall spaces of uncountable width. For example, it is not known whether there is in ZFC an $\left(\omega_{1}, \omega_{2}\right)$-LCS space. However, a $\left(\kappa, \kappa^{+}\right)$-LCS space for a specific regular cardinal $\kappa$ can be constructed by forcing. In this case, we have to use infinite forcing conditions and we do not need to use any special function.

If $(S, \leq)$ is a partial order and $s, t \in S$, we say that $\mathrm{s}, \mathrm{t}$ are compatible in $(S, \leq)$ if there is a $u \in S$ such that $u \leq s, t$.

Let $\kappa$ be an infinite cardinal. Put $T=\bigcup\left\{T_{\alpha}: \alpha<\kappa^{+}\right\}$where $T_{\alpha}=\{\alpha\} \times \kappa$ for $\alpha<\kappa^{+}$. We define $R_{\kappa}$ as the set of all $p=\left(x_{p}, \leq_{p}\right)$ such that:

(1) $x_{p} \in[T]^{<\kappa}$.

$(2) \leq_{p}$ is a partial ordering on $x_{p}$ such that for every $s, t \in x_{p}, s<_{p} t$ implies $\pi(s)<\pi(t)$.

(3) Every pair $s, t$ of compatible elements in $p$ has an infimum in $p$.

If $s, t \in x_{p}$ are compatible in $p$ and $v$ is the infimum of $s, t$ in $p$, we write $i_{p}\{s, t\}=$ $\{v\}$. If $s, t$ are incompatible in $p$, we write $i_{p}\{s, t\}=\emptyset$. Then, we define the partial ordering $\leq$ on $R_{\kappa}$ as we defined the partial ordering on $P_{\gamma}$, and we put $\mathbb{R}_{\kappa}=\left(R_{\kappa}, \leq\right)$. Also, we define the notion of a separated subset of $R_{\kappa}$ as above.

The proof of the following result simplifies the argument given in [13].

Theorem 6. If $\kappa^{<\kappa}=\kappa$, then $\mathbb{R}_{\kappa}$ is a cardinal-preserving partial order that forces the existence of $a\left(\kappa, \kappa^{+}\right)-L C S$ space. 
Proof. By an argument parallel to the one given in Proposition 2, we have that if $\mathbb{R}_{\kappa}$ preserves cardinals, then $\mathbb{R}_{\kappa}$ adjoins a $\left(\kappa, \kappa^{+}\right)$-LCS space. Clearly, $\mathbb{R}_{\kappa}$ is $\kappa$-closed. Then, in order to prove that $\mathbb{R}_{\kappa}$ is $\kappa^{+}$-c.c. consider a subset $D$ of $R_{\kappa}$ of size $\kappa^{+}$. Since $\kappa^{<\kappa}=\kappa$, we may assume that $D$ is separated. Let $x^{*}$ be the root of $\left\{x_{p}: p \in D\right\}$. We put $a^{*}=\pi\left[x^{*}\right]$ and $a_{p}=\pi\left[x_{p}\right]$ for $p \in D$. We have $\left|a_{p}\right|<\kappa$ for $p \in D$ and $|\beta \backslash \alpha| \leq \kappa$ for all $\alpha, \beta \in a^{*}$ with $\alpha<\beta$. Then as $\kappa^{<\kappa}=\kappa$, there is a subset $E$ of $D$ of size $\kappa^{+}$ such that $a^{*}$ is an initial segment of $a_{p}$ for every $p \in E$.

Take $p, q \in E$ such that if $\alpha \in a_{p} \backslash a^{*}$ and $\beta \in a_{q} \backslash a^{*}$, then $\alpha<\beta$. We have that $p$ and $q$ are compatible in $\mathbb{R}_{\kappa}$. For this, we define $r=\left(x_{r}, \leq_{r}\right)$ where $x_{r}=x_{p} \cup x_{q}$, and $s \leq_{r} t$ iff $s \leq_{p} t$ or $s \leq_{q} t$ or $\left(s \in x_{p} \backslash x^{*}, t \in x_{q} \backslash x^{*}\right.$ and $\left.h_{p q}(s) \leq_{q} t\right)$, where $h_{p q}$ denotes the adequate bijection between $p$ and $q$. It is easy to verify that $r$ is an element of $R_{\kappa}$ that extends $p$ and $q$.

In [14], by using the notion of a tree of intervals, it was proved that Theorem 6 can be extended to ordinals between $\kappa^{+}$and $\kappa^{++}$, and so we obtain the following result.

Theorem 7. If $\kappa$ is an infinite cardinal with $\kappa^{<\kappa}=\kappa$, there is a cardinal-preserving partial order that forces the existence of a $(\kappa, \alpha)$-LCS space for every $\alpha<\kappa^{++}$.

On the other hand, it was proved in [10] that thin-tall LCS spaces can be constructed from simplified morasses, which are combinatorial tools that permit us to construct a mathematical object of size $\kappa^{+}$from approximations of size smaller than $\kappa$. For the definition of this combinatorial notion, we refer the reader to [23].

Theorem 8. For every regular cardinal $\kappa$, the existence of a simplified $(\kappa, 1)$-morass implies the existence of a $\left(\kappa, \kappa^{+}\right)$-LCS space.

Sketch of proof. Let $\left\langle\left\langle\theta_{\xi}: \xi \leq \kappa\right\rangle,\left\langle\mathcal{F}_{\mu \xi}: \mu<\xi \leq \kappa\right\rangle\right\rangle$ be a simplified $(\kappa, 1)$-morass. Again, we construct the required space from an LCS structure. For $\xi \leq \kappa$ we construct an approximation $p_{\xi}=\left(x_{\xi}, \leq_{\xi}\right)$ of the desired LCS structure such that the following conditions are satisfied:

(1) For every $\xi<\kappa, p_{\xi}$ is an element of $R_{\kappa}$ with $\pi\left[x_{\xi}\right]=\theta_{\xi}$.

(2) $x_{\kappa}=\kappa^{+} \times \kappa$ and $\leq_{\kappa}$ is a partial ordering on $x_{\kappa}$.

(3) For every $\xi<\kappa$, if $\alpha, \beta \in \theta_{\xi}$ with $\alpha<\beta$ and $t \in x_{\xi} \cap T_{\beta}$, then there is an $\eta>\xi$ such that $(\alpha, \eta)<_{\xi}$.

Also, if $\xi<\eta \leq \kappa$ and $f \in \mathcal{F}_{\xi \eta}$, we consider a forcing condition $p_{\xi}^{f}=\left(x_{\xi}^{f}, \leq_{\xi}^{f}\right)$ isomorphic to $p_{\xi}$ such that $\pi\left[x_{\xi}^{f}\right]=f\left[\pi\left[x_{\xi}\right]\right]$ and for every $\nu<\kappa,(\alpha, \nu) \in x_{\xi}$ iff $(f(\alpha), \nu) \in x_{\xi}^{f}$. So, $p_{\xi}^{f}$ is just the projection of $p_{\xi}$ in the set of levels $f\left[\pi\left[x_{\xi}\right]\right]$.

The approximation $p_{0}$ is easy to construct. Suppose that $\xi=\mu+1$. Then, $\mathcal{F}_{\mu \xi}$ is a set formed by two functions $f$ and $g$ with a split point. We can amalgamate $p_{\mu}^{f}$ and $p_{\mu}^{g}$ proceeding as in the proof of Theorem 6 , and then we define the approximation 
$p_{\xi}$ from this amalgamation. And if $\xi$ is a limit, we define $p_{\xi}$ from the union of all the forcing conditions in $\left\{p_{\mu}^{f}: \mu<\xi, f \in \mathcal{F}_{\mu \xi}\right\}$.

If $\xi<\eta \leq \kappa$ and $f \in \mathcal{F}_{\xi \eta}$, we can verify that $x_{\eta} \supseteq x_{\xi}^{f}, \leq_{\eta} \uparrow x_{\xi}^{f}=\leq_{\xi}^{f}$ and $p_{\eta}$ preserves the infimums of pairs of compatible elements in $p_{\xi}^{f}$. Then, $p_{\kappa}$ gives rise to an LCS structure from which we can directly construct the desired $\left(\kappa, \kappa^{+}\right)$-LCS space.

The following result is an immediate consequence of Theorem 8.

Corollary 1. If $V=L$ holds, then there is a $\left(\kappa, \kappa^{+}\right)$-LCS space for every regular cardinal $\kappa$.

However, it is unknown whether $V=L$ implies that for every regular cardinal $\kappa$ and every ordinal $\alpha<\kappa^{++}$, there is a $(\kappa, \alpha)$-LCS space.

Also, it is known that if there is no inaccessible cardinal in $L$, then there is a simplified $(\kappa, 1)$-morass for every regular cardinal $\kappa$ (see [3]). But obviously, the non existence of inaccessible cardinals in ZFC would imply the non existence of inaccessible cardinals in $L$. So, we obtain from Theorem 8 the following result.

Corollary 2. If there is no inaccessible cardinal in $Z F C$, then there is a $\left(\kappa, \kappa^{+}\right)-L C S$ space for every regular cardinal $\kappa$.

It is not known whether the above existence results are true for singular cardinals.

Also, we want to remark that it has recently been proved in [7] that there is in ZFC an LCS space $X$ such that ht $(X)=\omega_{2}$ and $\left|I_{0}(X)\right|=\omega_{1}$.

\section{Acknowledgements}

I want to express my gratitude to Stevo Todorčević for his encouragement to write this paper.

\section{References}

[1] J.E.Baumgartner and S.Shelah, Remarks on superatomic Boolean algebras, Annals of Pure and Applied Logic 33 (1987), 109-129.

[2] M.Bekkali, Topics in Set Theory, Lecture Notes in Mathematics 1476, Springer-Verlag, Berlin, 1991.

[3] K.J.Devlin, Constructibility, Springer-Verlag, Berlin, 1984.

[4] A.Dow and P.Simon, Thin-tall Boolean algebras and their automorphism groups, Algebra Universalis 29 (1992), 211-226.

[5] R.Engelking, General Topology, Heldermann Verlag, Berlin, 1989.

[6] T.Jech, Set Theory, Academic Press, New York, 1978.

[7] I. Juhász, S.Shelah, L.Soukup and Z.Szentmiklóssy, A tall space with a small bottom, Proceedings of the American Mathematical Society (to appear). 
[8] I.Juhász and W.Weiss, On thin-tall scattered spaces, Colloquium Mathematicum 40 (1978), 63-68.

[9] W.Just, Two consistency results concerning thin-tall Boolean algebras, Algebra Universalis 20 (1985), 135-142.

[10] P.Koepke and J.C.Martínez, Superatomic Boolean algebras constructed from morasses, The Journal of Symbolic Logic 60 (1995), 940-951.

[11] S.Koppelberg, General theory of Boolean algebras, Handbook of Boolean algebras, Volume 1 (J.D.Monk and R.Bonnet,editors), North-Holland, Amsterdam, 1989.

[12] K.Kunen, Set Theory, North-Holland, Amsterdam, 1980.

[13] J.C.Martínez, A consistency result on thin-tall superatomic Boolean algebras, Proceedings of the American Mathematical Society 115 (1992), 473-477.

[14] J.C.Martínez, A forcing construction of thin-tall Boolean algebras, Fundamenta Mathematicae 159 (1999), 99-113.

[15] J.C.Martínez, A consistency result on thin-very tall Boolean algebras, Israel Journal of Mathematics 123 (2001), 273-284.

[16] M.Rajagopalan, A chain-compact space which is not strongly scattered, Israel Journal of Mathematics 23 (1976), 117-125.

[17] J.Roitman, Height and width of superatomic Boolean algebras, Proceedings of the American Mathematical Society 94 (1985), 9-14.

[18] J.Roitman, Superatomic Boolean algebras, Handbook of Boolean algebras, Volume 3 (J.D.Monk and R.Bonnet,editors), North-Holland, Amsterdam, 1989, pp. 719-740.

[19] J.Ruyle, Cardinal sequences of PCF structures, Doctoral dissertation, University of California, Riverside, 1998.

[20] S.Shelah, Cardinal arithmetic, Oxford University Press, 1995.

[21] S.Shelah, On what I do not understand (and have something to say), Part I, Fundamenta Mathematicae 166 (2000), 1-82.

[22] S.Todorčević, Partitioning pairs of countable ordinals, Acta Mathematica 159 (1987), 261-294.

[23] D.J.Velleman, Simplified morasses, The Journal of Symbolic Logic 49 (1984), 257-271. 